\title{
Mental Rotation Meets the Motion Aftereffect: The Role of hV5/MT + in Visual Mental Imagery
}

\author{
Ruth Seurinck ${ }^{1}$, Floris P. de Lange ${ }^{2}$, Erik Achten ${ }^{1}$, \\ and Guy Vingerhoets ${ }^{1}$
}

\begin{abstract}
A growing number of studies show that visual mental imagery recruits the same brain areas as visual perception. Although the necessity of hV5/MT + for motion perception has been revealed by means of TMS, its relevance for motion imagery remains unclear. We induced a direction-selective adaptation in hV5/MT + by means of an MAE while subjects performed a mental rotation task that elicits imagined motion. We concurrently measured behavioral performance and neural activity with fMRI, enabling us to directly assess the effect of a perturbation
\end{abstract}

\section{INTRODUCTION}

For most people, visual mental imagery is a familiar aspect of everyday experience. It is often referred to as "visualizing," "picturing," or "seeing with the mind's eye." A growing number of studies show that visual mental imagery recruits the same brain areas as visual perception, implying that the underlying representations of such mental transformations are depictive or picture-like instead of symbolic or language-like (Kosslyn, 1994). It has been demonstrated that visual imagery is associated with activity in retinotopically organized early visual areas (Klein et al., 2004). An fMRI study also observed imagery-related activity in the higher order visual area of hV5/MT+ involved in motion perception, suggesting that subjects can to some extent reproduce visual motion during mental imagery (Slotnick, Thompson, \& Kosslyn, 2005).

Although the functional relevance of hV5/MT + for perception of (physically present) motion is unequivocal (d'Alfonso et al., 2002; Hotson \& Anand, 1999; Anand, Olson, \& Hotson, 1998; Walsh, Ellison, Battelli, \& Cowey, 1998; Beckers \& Zeki, 1995; Newsome \& Pare, 1988), the necessity of this region for motion imagery has never been assessed. In the present study, we addressed this question by combining in fMRI (1) mental rotation, an established visual mental imagery paradigm that implies imagery of motion, with (2) a localized perturbation in the form of differential adaptation of direction-selective neurons in hV5/MT+ or direction-

${ }^{1}$ Ghent University, Belgium, ${ }^{2}$ Radboud University Nijmegen, the Netherlands of hV5/MT+ on other cortical areas involved in the mental rotation task. The activity in hV5/MT+ increased as more mental rotation was required, and the perturbation of hV5/MT + affected behavioral performance as well as the neural activity in this area. Moreover, several regions in the posterior parietal cortex were also affected by this perturbation. Our results show that $\mathrm{hV} 5 / \mathrm{MT}+$ is required for imagined visual motion and engages in an interaction with parietal cortex during this cognitive process.

selective adaptation known to cause an MAE. In the classic paradigm of mental rotation, subjects are required to make comparative decisions about rotated objects (Shepard \& Metzler, 1971). Response times usually increase monotonically with increasing angular disparity of the object, suggesting that subjects rotate a mental image of the object (Shepard \& Metzler, 1971). The MAE is a perceptual illusion where prolonged viewing of motion in a certain direction elicits perception of illusory motion in the opposite direction when a subsequent stationary stimulus is presented. Neurophysiological recordings suggest that the MAE is the result of an imbalance in the postadaptation responsiveness of different subpopulations of direction-selective neurons in area hV5/ MT+ (Van Wezel \& Britten, 2002).

The combination of an MAE and mental rotation has been selected to study the functional relevance of hV5/MT+ in visual mental imagery for several reasons. The characteristic behavioral profile of mental rotation provides a measurement for task performance, making the inner phenomenon of mental imagery more accessible for scientific research.

On the one hand, there is previous behavioral evidence that an MAE influences task performance during mental rotation: An MAE that is congruent with the direction of the imagined motion of the mental rotation task leads to relatively shorter RTs, whereas an MAE that obstructs the imagined motion results in relatively longer RTs (Heil, Bajric, Rosler, \& Hennighausen, 1997). However, the neural mechanism of this influence has not been elucidated.

On the other hand, neuroimaging studies have generally found a network of parietal and frontal regions to be involved in mental rotation (Mourao-Miranda, Ecker, Sato, 
\& Brammer, 2009; Zacks, 2008; Ecker, Brammer, David, \& Williams, 2006; Wolbers, Schoell, \& Buchel, 2006; Richter et al., 2000; Parsons et al., 1995) as well as hV5/MT+ (de Lange, Hagoort, \& Toni, 2005). Yet, it is impossible on the basis of these neuroimaging studies to distinguish cortical areas that are necessary for motion imagery from corollary activations. By simultaneously registering the effects of manipulating hV5/MT + on both the task performance and the neural activity of an established visual mental imagery task, we aimed to directly test the involvement of $\mathrm{hV} 5 / \mathrm{MT}+$ and the neural consequences of its perturbation during motion imagery.

We expected that neural activity in hV5/MT+ increases with angular disparity of the stimuli, reflecting the imagined motion. Furthermore, we expected that an obstructing MAE leads to longer RTs and increased neural activity in hV5/MT+. Finally, we hypothesized that if hV5/MT+ interacts with parietal cortex to perform the imagery task, an obstructing MAE will result not only in increased activation in hV5/MT + but also in parietal regions involved in mental rotation.

\section{METHODS}

\section{Participants}

Following written informed consent according to the institutional guidelines of the Ethics Committee of the Ghent University Hospital, 16 healthy male volunteers participated in the study. All participants were right-handed and were between 21 and 28 years old (mean $=24.0$ years, $S D=2.0$ years). Participants were graduate students or had an equal level of education.

\section{Experimental Design and Procedure}

Each trial started with the presentation of a fixation cross for a variable interval between 2.5 and $3.5 \mathrm{sec}$. This was followed by an MAE induction phase, during which a sinusoidal grating was presented for 5 sec that rotated either clockwise or counterclockwise at a velocity of $121 \mathrm{deg} / \mathrm{sec}$ (see Figure 1). Both the velocity and the duration were selected on the basis of pilot data. Immediately after the induction phase, the task stimulus was presented for a duration of 2 sec.

A series of five different alphanumeric stimuli were selected for the mental rotation task (2, R, a, t, and $\mathrm{k}$ ). They were presented in either their canonical form or mirrored across the vertical axis. Each stimulus was shown at $45^{\circ}$, $80^{\circ}, 115^{\circ}$, or $150^{\circ}$ disparity from the upright position in either clockwise or counterclockwise orientation. Participants were required to make a canonical/mirror judgment by pressing an MRI-compatible button box with the index/ middle finger of their right hand. The RT cutoff was $2 \mathrm{sec}$. All mental rotation stimuli were presented four times: twice when the direction of the mental rotation and the MAE were congruent and twice when these were incongruent,

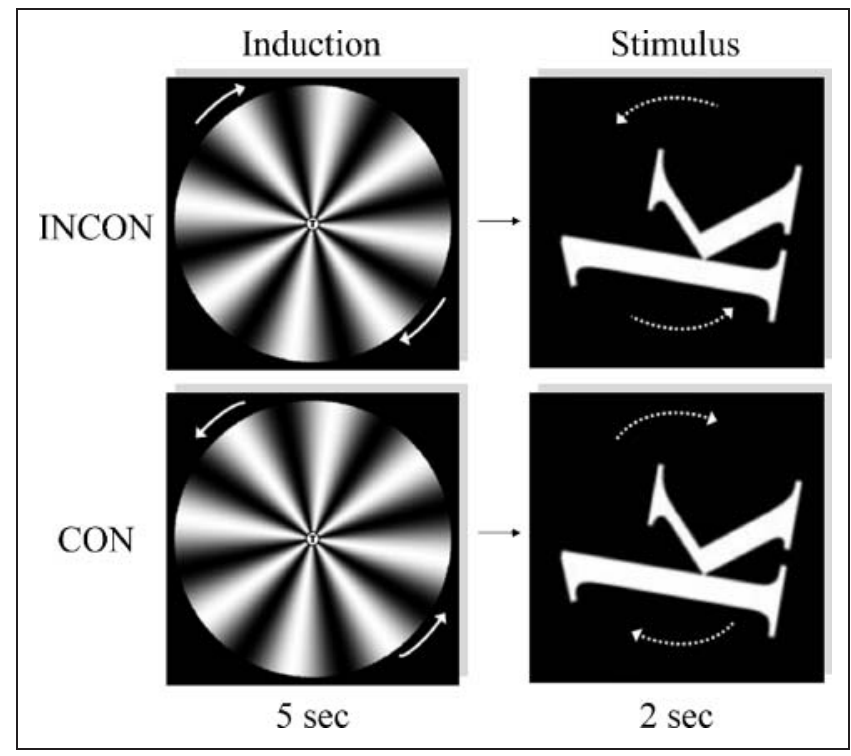

Figure 1. Timeline of two trial examples where the mental rotation task is immediately preceded by a unidirectional rotating sinusoidal grating to induce an MAE, resulting in illusory motion of the stimulus. Although the mental rotation stimulus and task are identical in both trials, the upper example is an incongruent (INCON) trial because the represented direction of the MAE is opposite to the direction of the required clockwise mental rotation, whereas the lower example is a congruent $(\mathrm{CON})$ trial where these directions are the same.

resulting in congruent (CON) and incongruent (INCON) trials. This led to a total number of 320 trials. To maximize the MAE, trials were grouped in blocks of 10 according to the direction of the MAE. The blocks were presented alternately and separated by a low-level baseline block in which subjects looked at a fixation cross for $12 \mathrm{sec}$. Note that although the MAE was blocked into groups of 10 trials, the MAE congruency varied from trial to trial because each block consisted of stimuli in both clockwise and counterclockwise orientation.

Finally, to ensure attention and fixation throughout the induction phase, an oddball task was inserted. The sinusoidal grating contained a small $\mathrm{T}$ in the center of the grating, which flipped upside down from its canonical position in $10 \%$ of the trials. Participants were instructed to monitor this central stimulus and to indicate a change as soon as they perceived it with a button press.

The number of trials was spread out over three separate runs, leading to a total measuring time of 1 hour. All participants were familiarized with the task before scanning. Immediately before the experiment, they performed two practice blocks of 10 trials in the scanner.

We additionally included a passive viewing task in the experiment to localize regions that were responsive to the MAE. During the MAE condition, the sinusoidal grating rotated clockwise or counterclockwise at constant velocity for a duration of $30 \mathrm{sec}$. During the control condition, the grating changed its direction of rotation every $0.5 \mathrm{sec}$. At the end of each induction phase, the sinusoidal grating 
stopped and remained stationary for $30 \mathrm{sec}$ to create a test phase for the MAE. The localizer task contained six blocks, each comprising an alternating induction phase and a unidirectional induction phase with their respective test phases. In one subject, only five blocks were collected because of technical problems. The blocks were preceded by a fixation cross for $15 \pm 1 \mathrm{sec}$, and within the different blocks, the order of the control task and the MAE was counterbalanced.

Stimulus presentation and response collection was controlled using E-Prime (Psychology Software Tools, Pittsburgh, PA) and delivered within the magnet by means of MRI-compatible goggles (VisuaStim XGA; Resonance Technology, Northridge, CA).

\section{Scanning Procedure}

Scanning was performed at $3 \mathrm{~T}$ on a Siemens TRIO MR scanner (Siemens Medical Systems, Erlangen, Germany) using an eight-channel PA head coil for radio-frequency transmission and signal reception. A 3-D high-resolution T1-anatomical image of the whole brain (3-D MPRAGE, 176 slices, slice thickness $=0.9$, in-plane resolution $=$ $0.9 \times 0.9$, repetition time $[\mathrm{TR}]=1550 \mathrm{msec}$, echo time $=$ 2.89) was acquired for coregistration with the functional images. Finally, a total of 2270 functional EPI images in four separate runs (experiment: 635, 635, and 575; localizer: 425) were obtained during stimulus presentation for each participant $(\mathrm{TR}=1940 \mathrm{msec}$, echo time $=35 \mathrm{msec}$, flip angle $=$ $80^{\circ}, 28$ slices, slice thickness $=3 \mathrm{~mm}$ with a distance factor of $17 \%$, field of view $=224 \mathrm{~mm}$, matrix $=64 \times 64$, resulting in an isotropic voxel size of $3.5 \times 3.5 \times 3.5 \mathrm{~mm}$ ).

\section{Image Analysis}

Image analysis was conducted with statistical parametric mapping (SPM5) (www.fil.ion.ucl.ac.uk/spm). To remove motion artifacts, all functional images were first realigned to the mean image using a rigid body spatial transformation (Friston et al., 1995). Next, the slices of each functional image were temporally realigned with the acquisition time of the middle slice. The resulting images were normalized to a standard EPI template in the Montreal Neurological Institute stereotaxic space and resampled at an isotropic voxel size of $2 \mathrm{~mm}$ by using an affine transformation followed by a nonlinear transformation. Finally, the normalized images were smoothed with an isotropic 8-mm FWHM Gaussian kernel. The anatomical images of the participants were spatially coregistered with their corresponding mean functional image and subsequently spatially normalized using the same parameters that were applied for the normalization of the functional images.

The statistical model for the main experiment was constructed for each participant on a trial-by-trial basis, including separate events for the induction phase and the mental rotation task. The variance in neural signal during the mental rotation task was modeled along two orthogonal dimensions: a main effect of MAE congruency and a modulation by angular disparity (four levels: $45^{\circ}$ to $150^{\circ}$, in $35^{\circ}$ steps). The main effect of MAE congruency was represented by congruent events (CONm) and incongruent MAE events (INCONm). A linear polynomial expansion was used to model the neural modulation of angular disparity or rotation-related activity for these events (CONp and INCONp). The hemodynamic response for each event was modeled by convolving boxcars, time locked to their occurrence, with a canonical hemodynamic response function to form covariates in a general linear model (Friston et al., 1994). The marginal mean RT per session, except oddball and error trials, defined the length of the boxcars for the mental rotation events. Other sources of variance included in the statistical model as covariates of no interest were oddball trials, error trials, movement-related effects, low-frequency signal drifts over time, and overall differences between sessions. Parameters for each covariate were estimated by a least squares fit to the data.

Linear contrasts of these parameter estimates, averaged across sessions, constituted the data for the second-stage analysis. In this analysis, we tested for the reliability of the neural response in one-sample $t$ tests, treating subjects as a random variable (Friston, Holmes, Price, Buchel, \& Worsley, 1999). Because this is a random effects group analysis, the inferences we report pertain to the average size of the effect in the population from which the subjects were drawn (Friston, Holmes, \& Worsley, 1999).

The contrast CONp $\cap$ INCONp representing the common angular disparity-related activity was used to identify the common rotation-related activity or the mental rotation network. Because we used a mass univariate approach, the inference introduces a multiple comparisons problem. The family-wise error (FWE) correction was used to correct for having performed multiple tests. The eventual inferences pertain to the voxel level and are based on a corrected threshold of $p<.05$ FWE corrected.

To test for the modulation of the mental rotation network by the MAE congruency, we defined the following contrasts: $\mathrm{CONm}>\mathrm{INCONm}$, INCONm > CONm, CONp > INCONp, and INCONp $>$ CONp. Because we focused our search on regions that modulated their activity as a function of mental rotation (i.e., the mental rotation network), we could enhance our statistical sensitivity by correcting for multiple comparisons in a reduced search space defined by the (orthogonally defined) mental rotation network (Friston, Rotshtein, Geng, Sterzer, \& Henson, 2006). We used the stereotaxic coordinates of the mental rotation network nodes to position the ROIs on our $\operatorname{SPM}(t) \mathrm{s}$, and their radius was defined by the amount of smoothing that was applied to the images $(8 \mathrm{~mm})$ and corrected our results for multiple comparisons using an FWE threshold of $p<.05$ within this search volume (Worsley et al., 1996).

A similar procedure was used for the analysis of the localizer experiment. The statistical model comprised two 
events: one for the alternating and one for the unidirectional induction phase, modeled by convolving boxcars with a canonical hemodynamic response. The duration of the boxcar was set to $35 \mathrm{sec}$ instead of $30 \mathrm{sec}$ to include the additional experience of visual motion brought on by the MAE. A conjunction analysis of both types of induction was used to identify in every subject the peak voxel in each hemisphere that responded maximally to the rotating grating. A time course analysis in each peak voxel was conducted using MarsBaR (Brett, Anton, Valabregue, $\&$ Poline, 2002). First, the mean time course of activity was extracted from an ROI positioned on the peak voxel with a radius of $8 \mathrm{~mm}$. Next, the peristimulus time histogram for both types of induction was calculated by means of a finite impulse response model in that the length of each time bin equaled one TR. A total of 30 bins were used, encompassing a period of $58.2 \mathrm{sec}$ time locked to the start of the induction phase. Finally, to directly compare the two obtained time courses per ROI, a paired $t$ test was conducted for each time bin.

To identify the corresponding anatomical regions of significant signal change, the SPM $(t)$ s were superimposed on the high-resolution anatomical scan of each subject, and the anatomical details were compared with the atlas of Duvernoy, Cabanis, and Vannson (1991).

\section{Behavioral Analysis}

The mean RTs and error rates (ERs) registered during the scanning sessions were modeled by a $2 \times 4$ repeated measures ANOVA with respective main effects MAE congruency and Angular disparity. The alpha level was set at $p<.05$, Greenhouse-Geisser corrected where required. Analogous to the imaging analysis, oddball trials were not included. For the RT analysis, only correct trials were considered.

\section{RESULTS}

\section{Behavioral Performance}

Both RTs and ERs increased with increasing angular disparity-RT, $F(1.64,24.55)=107.00, p<.001$; ER, $F(1.43,21.51)=17.46, p<.001-$ showing both linearRT, $F(1,15)=150.16, p<.001 ; \mathrm{ER}, F(1,15)=20.58$, $p<.001-$ and quadratic trends, RT, $F(1,15)=27.39$, $p<.001 ; \mathrm{ER}, F(1,15)=9.38, p=.008$.

As revealed in Figure 2A, subjects responded overall slower during incongruent trials, $F(1,15)=16.32, p=$ .001. Although the difference in RT between congruent and incongruent trials increased when the largest mental rotation of $150^{\circ}$ was required, the interaction between Angular disparity and MAE congruency only showed a trend toward significance, $F(3,45)=2.70, p=.057$. Subjects were not overall more error prone during incongruent trials, $F(1,15)=3.00, p=.10$, but there was a

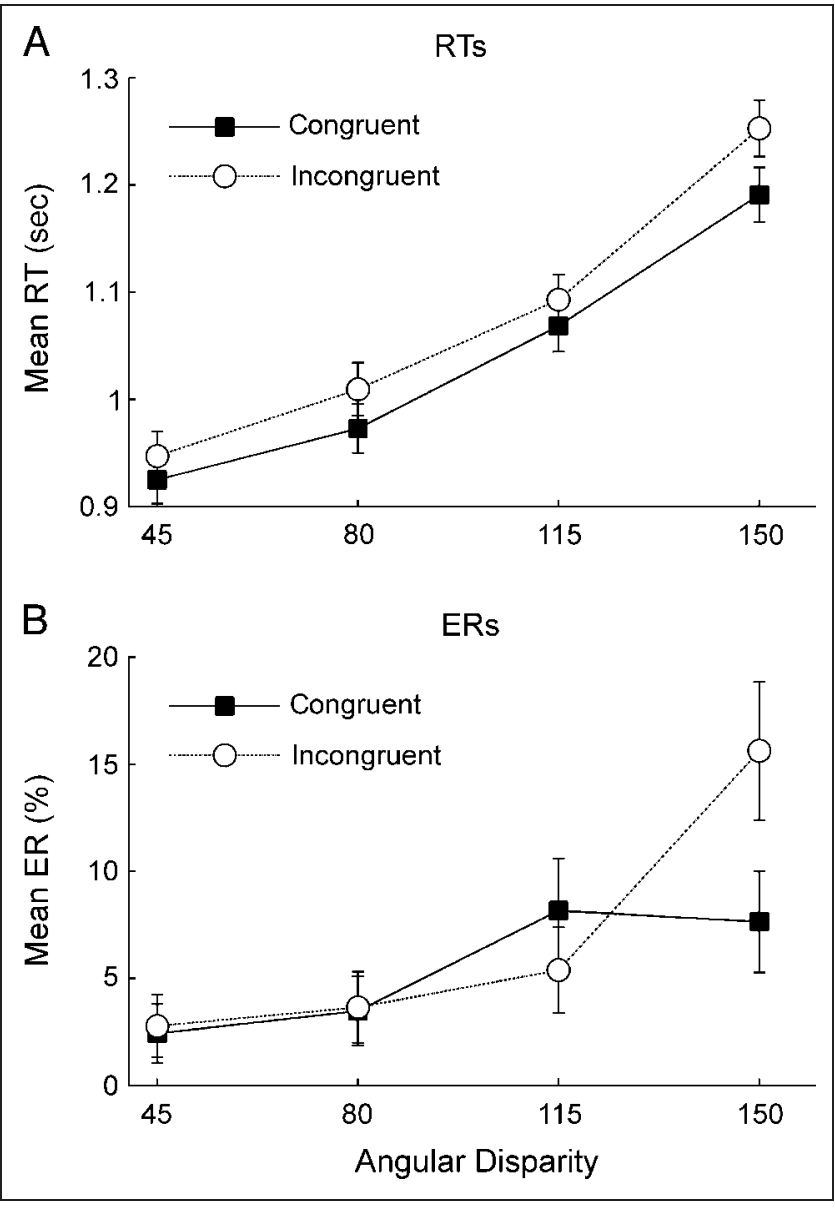

Figure 2. Behavioral performance represented by the mean RTs (A) and mean ERs (B) and their 95\% confidence intervals as a function of angular disparity. For both the congruent and the incongruent trials, the ERs and the RTs increase with angular disparity. Subjects are consistently slower during the incongruent than the congruent trials. Only at the largest angular disparity of $150^{\circ}$ are they also more error prone during the incongruent trials.

significant interaction with angular disparity, $F(3,45)=$ $13.23, p<.001$. Figure $2 \mathrm{~B}$ shows that only at the largest angular disparity of $150^{\circ}$ subjects made significantly more errors when the direction of the MAE was incongruent with the mental rotation direction.

\section{Common Rotation-related Activity: The Mental Rotation Network}

There was a linear increase in neural activity with increasing angular disparity in a network of visual, parietal, and frontal areas, that is, the extrastriate visual cortex, the various segments along the intraparietal sulcus (IPS), the superior and inferior frontal gyrus, and the anterior cingulate sulcus (see Table 1, Figure 3).

The activity found in the left temporo-occipital fissure centered on the ascending limb of the inferior temporal sulcus corresponds to hV5/MT+, as demonstrated in previous studies on the human homologue of V5/MT (Dumoulin 
Table 1. Common Angular Disparity-related Activity (CONp $\cap$ INCONp) or the Mental Rotation Network

\begin{tabular}{|c|c|c|c|c|c|c|}
\hline \multirow{2}{*}{$\frac{\text { Anatomical Region }}{\text { Temporo-occipital fissure (hV5/MT+) }}$} & \multirow{2}{*}{$\frac{\text { Cluster Size }}{10}$} & \multirow{2}{*}{$\frac{\text { Hemisphere }}{\mathrm{L}}$} & \multirow{2}{*}{$\frac{Z \text { Score }}{5.33}$} & \multicolumn{3}{|c|}{ Stereotaxic Coordinates } \\
\hline & & & & -40 & -78 & 0 \\
\hline Temporo-occipital fissure (hV5/MT+) & 63 & $\mathrm{~L}$ & 5.32 & -48 & -72 & -6 \\
\hline Transverse occipital sulcus & 31 & $\mathrm{~L}$ & 5.02 & -26 & -94 & 12 \\
\hline Posterior segment IPS (pIPS) & 49 & $\mathrm{~L}$ & 5.59 & -24 & -78 & 36 \\
\hline Posterior segment IPS (pIPS) & 2 & $\mathrm{~L}$ & 5.09 & -20 & -76 & 46 \\
\hline Posterior segment IPS (pIPS) & 3 & $\mathrm{R}$ & 5.05 & 24 & -72 & 48 \\
\hline Precuneus & 288 & $\mathrm{~L}$ & 6.21 & -16 & -64 & 52 \\
\hline Precuneus & 230 & $\mathrm{R}$ & 5.87 & 16 & -66 & 50 \\
\hline Middle segment IPS (mIPS) & 247 & $\mathrm{~L}$ & 5.83 & -30 & -54 & 62 \\
\hline Middle segment IPS (mIPS) & 230 & $\mathrm{R}$ & 5.31 & 28 & -56 & 54 \\
\hline Anterior segment IPS (aIPS) & 247 & $\mathrm{~L}$ & 5.31 & -40 & -38 & 46 \\
\hline Anterior segment IPS (aIPS) & 52 & $\mathrm{R}$ & 5.44 & 34 & -40 & 46 \\
\hline Superior frontal sulcus (dorsolateral premotor cortex) & 240 & $\mathrm{~L}$ & 6.02 & -24 & -4 & 58 \\
\hline Superior frontal sulcus (dorsolateral premotor cortex) & 61 & $\mathrm{R}$ & 5.86 & 30 & -6 & 56 \\
\hline Inferior frontal gyrus & 7 & $\mathrm{R}$ & 5.16 & 40 & 6 & 34 \\
\hline Anterior cingulate sulcus & 29 & $\mathrm{~L}$ & 5.51 & -10 & 20 & 42 \\
\hline
\end{tabular}

et al., 2000; Tootell et al., 1995; Watson et al., 1993) in combination with related satellite areas (Sunaert, Van Hecke, Marchal, \& Orban, 1999). The results of the localizer experiment confirm this. The mean $\pm S D$ coordinate of the individually defined peak voxels in the left hemisphere that responded maximally to the rotary motion of the induction phase was $-43 \pm 4,-74 \pm 5$, and $6 \pm 5$. In addition, an ROI analysis positioned on this mean voxel with a radius of $8 \mathrm{~mm}$ also revealed common rotationrelated activity in the main experiment.

The bilateral neural activity in the superior frontal sulcus can be situated within the boundaries of dorsolateral premotor cortex (Mayka, Corcos, Leurgans, \& Vaillancourt, 2006) and is compatible with the site of the FEFs (Amiez, Kostopoulos, Champod, \& Petrides, 2006; Berman et al., 1999).

Three different bilateral areas can be clearly distinguished along the IPS, positioned in respectively a posterior segment of IPS (pIPS), a middle segment of IPS (mIPS), and an anterior segment of IPS (aIPS). The neural activity in pIPS near the parieto-occipital sulcus is more pronounced in the left hemisphere with an additional activation, located immediately inferior to the bilateral recruited pIPS. This cortical area is positioned immediately laterally $(>12 \mathrm{~mm}$ ) of V6 (Pitzalis et al., 2006) and inferior to the orientation selective CIP (Shikata et al., 2001, 2003). The brain area mIPS lies close to the human parietal eye fields (PEF) (Koyama et al., 2004; Berman et al., 1999). In the vicinity of aIPS, both a polymodal motion-sensitive area known as the ventral intraparietal (VIP; Bremmer et al., 2001) and the anterior intraparietal associated with tactile and visual object processing (Grefkes \& Fink, 2005) have been situated.

\section{Differential Rotation-related Activity: The Effect of MAE Congruency}

There was larger rotation-related activity in the left hV5/ $\mathrm{MT}+$ when the MAE was incongruent compared with a congruent MAE. The time course analysis of the localizer experiment revealed an MAE effect in the individually defined areas corresponding to left hV5/MT + . Figure 4 clearly shows that the percent signal change was higher in the MAE compared with the control condition in several consecutive measurement points after the grating became stationary: 4th scan, $t(15)=3.00, p=.009 ; 5$ th scan, $t(15)=$ $3.77, p=.002$; 6th scan, $t(15)=3.28, p=.005 ; 7$ th scan, $t(15)=2.20, p=.044$; 9 th scan, $t(15)=3.05, p=.008$; 10th scan, $t(15)=2.48, p=.026$. Furthermore, an additional ROI analysis demonstrated in these same individually defined areas corresponding to left $\mathrm{hV} 5 / \mathrm{MT}+$, just as in the group results, larger rotation-related activity during an incongruent MAE than during a congruent MAE, $t(15)=2.72$, $p=.016$.

Several other areas of the mental rotation network showed a modulation of MAE congruency. These areas were all located in the parietal lobe, that is, the inferior left pIPS, the right $\mathrm{mIPS}$, the right precuneus, and the right aIPS. (see Table 2, Figure 5). To exclude that the perturbation has a main effect on any of these parietal areas, we conducted a set of additional time course analyses to verify if any of these 
areas showed an MAE in itself. Using the data from the localizer experiment, we extracted the time course in ROIs corresponding to the abovementioned parietal areas in the mental rotation network that showed a modulation of MAE congruency. The ROIs were created by positioning a sphere with a

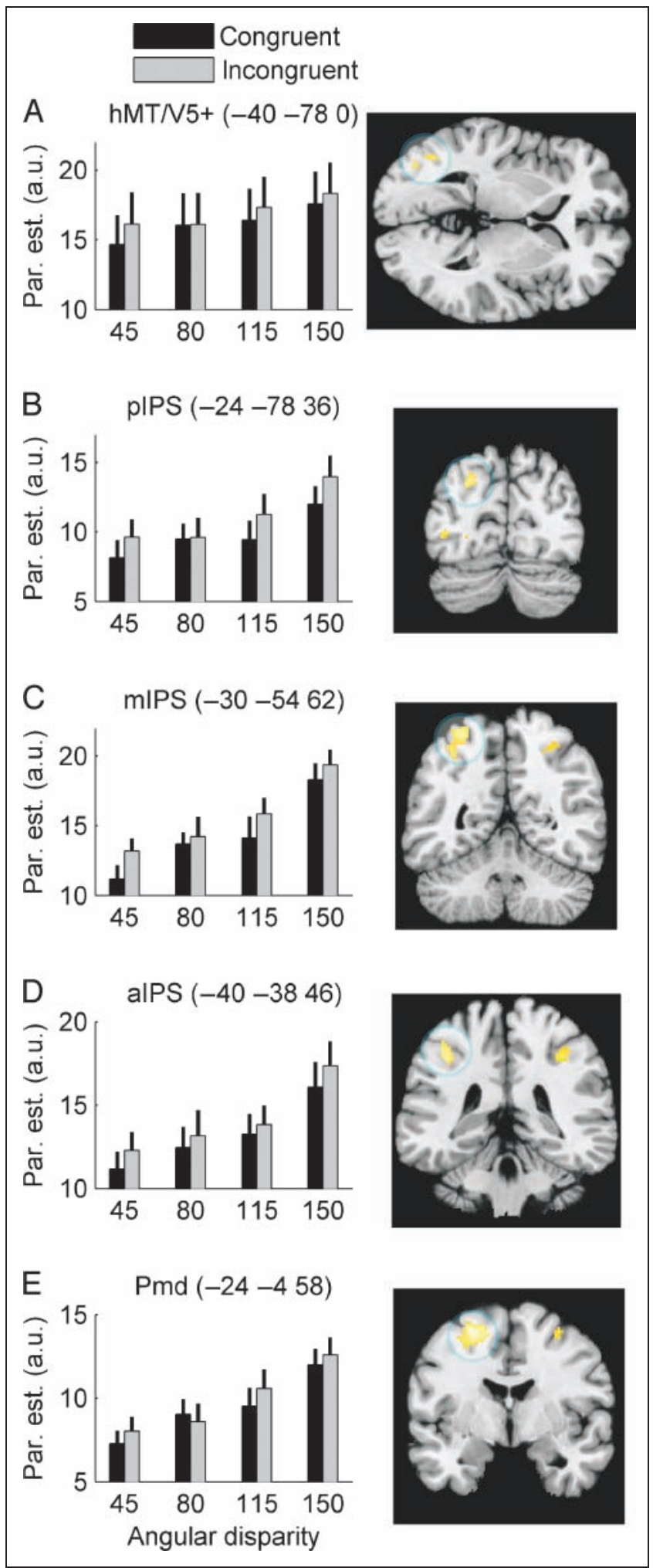

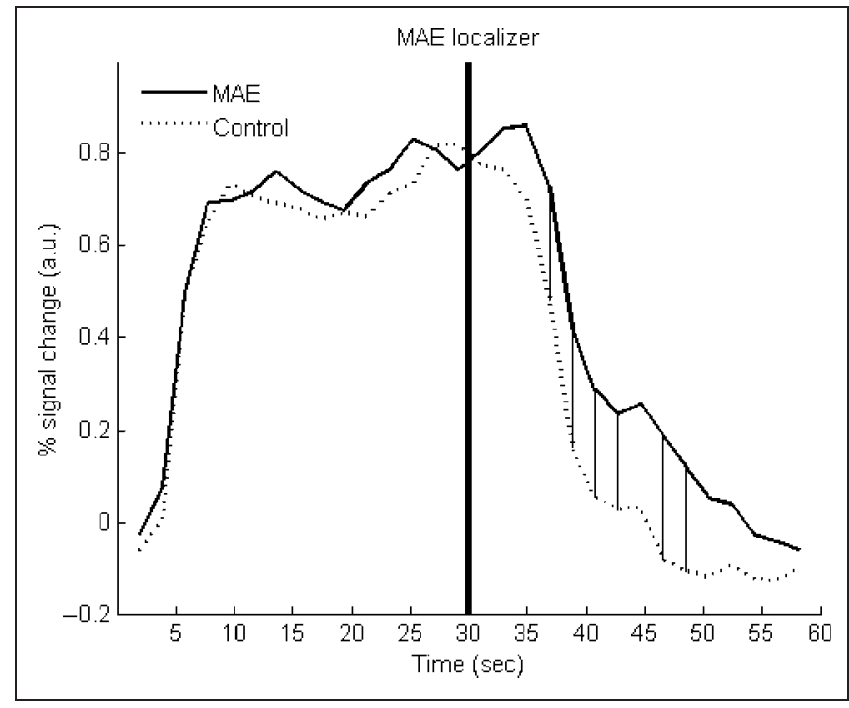

Figure 4. Time course analysis of the MAE localizer experiment. A unidirectional (MAE) or alternating (Control) induction phase of $30 \mathrm{sec}$ is followed by a stationary grating. The vertical drop lines between the two curves indicate measurement points where the difference in percent signal change between the two conditions is significant.

radius of $8 \mathrm{~mm}$ on the peak voxel of each of these parietal areas. In contrast to left $\mathrm{hV} 5 / \mathrm{MT}+$, none of these parietal areas demonstrated an MAE effect in the localizer experiment.

Finally, there were no areas that showed greater rotationrelated activity during congruent MAE than during an incongruent MAE.

\section{DISCUSSION}

In this study, we tested for the functional relevance of hV5/MT + during visual mental imagery by examining the behavioral performance and neural activity in a mental rotation task after perturbation of hV5/MT + with an MAE. We observed that an MAE that was incongruent with the direction of mental rotation slowed down behavioral performance and led to increased activity in hV5/ $\mathrm{MT}+$. Furthermore, several regions in the parietal cortex that were involved in the mental rotation process were also affected by MAE congruency. However, in contrast

Figure 3. A selection of left hemispheric brain areas in which the neural activity increases linearly with angular disparity (for a full listing, see Table 1). The overlays of the brain areas on a T1-weighted Montreal Neurological Institute single subject template (Eickhoff et al., 2005), shown in the right column, are accompanied in the left column by the corresponding parameter estimates (mean + SEM, arbitrary units) for the congruent and incongruent trials as a function of angular disparity. The presented cortical regions accompanied by their stereotaxic coordinates are hV5/MT+ (A), posterior segment IPS (B), middle segment IPS (C), anterior segment IPS (D), and dorsolateral premotor cortex (Pmd) (E). The parameter estimates clearly show an increase in signal change with angular disparity and are usually higher in the incongruent trials, except for left Pmd. 
Table 2. Differential Angular Disparity-related Activity (INCONm > CONm) or the Nodes of the Mental Rotation Network That Are Modulated by MAE Congruency

\begin{tabular}{|c|c|c|c|c|c|}
\hline \multirow{2}{*}{$\frac{\text { Anatomical Region }}{\text { Temporo-occipital fissure (hV5/MT+) }}$} & \multirow{2}{*}{$\frac{\text { Hemisphere }}{\mathrm{L}}$} & \multirow{2}{*}{$\frac{Z \text { Score }}{3.20}$} & \multicolumn{3}{|c|}{ Stereotaxic Coordinates } \\
\hline & & & -34 & -76 & -4 \\
\hline Temporo-occipital fissure (hV5/MT+) & $\mathrm{L}$ & 3.17 & -46 & -68 & -12 \\
\hline Posterior segment IPS (pIPS) & $\mathrm{L}$ & 3.48 & -20 & -82 & 32 \\
\hline Precuneus & $\mathrm{R}$ & 3.21 & 14 & -64 & 64 \\
\hline Middle segment IPS (mIPS) & $\mathrm{R}$ & 3.15 & 22 & -48 & 52 \\
\hline Anterior segment IPS (aIPS) & $\mathrm{R}$ & 3.26 & 34 & -38 & 40 \\
\hline
\end{tabular}

to hMT/V5+, none of these parietal areas showed a main effect of MAE as revealed by a localizer experiment. This suggests that hV5/MT + is functionally relevant during mental imagery of visual motion and engages in functional interaction with parietal cortex. Below, we detail and interpret these behavioral and cerebral effects.

\section{Behavioral Performance}

We found that MAE influenced task performance during mental rotation: An MAE that was congruent with the di-

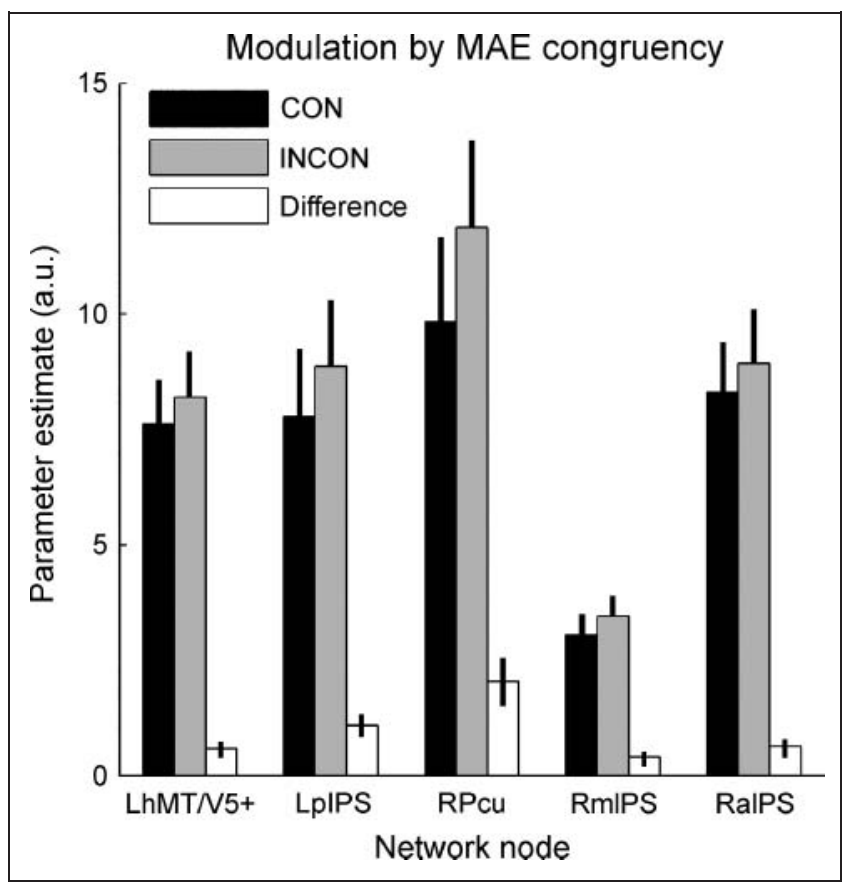

Figure 5. Mental rotation network nodes that are modulated by MAE congruency or brain areas that show both common and differential rotation-related activity. The parameter estimates (mean + SEM, arbitrary units) for the congruent and incongruent trials are accompanied by the relative difference (INCONm $>$ CONm: mean \pm 95\% CI, arbitrary units). Besides left hV5/MT+ $(-34,-76,-4)$, also a number of parietal areas show differential rotation-related activity: left pIPS (-20, -82, 32), right precuneus (Pcu) $(14,-64,64)$, right mIPS $(22,-48,52)$, and right aIPS $(34,-38,40)$. rection of the imagined motion of the mental rotation task lead to relatively shorter RTs, whereas an MAE that obstructed the imagined motion lead to relatively longer RTs. These findings are consistent with an earlier study (Heil et al., 1997) and indicate that our experimental manipulation was successful.

\section{Neural Activity Related to Visual Mental Imagery}

To isolate neural activity related to visual mental imagery of motion or the imagined transformation process from other processes in the mental rotation task such as visual stimulus encoding and motor response, we assessed that areas showed an increase in neural activity with increasing angular disparity. Cortical areas showing this modulation of activity included several regions of the parietal and frontal cortex and hV5/MT + . The involvement of these areas during the transformation of visual mental images is in accordance with the results of previous studies (de Lange et al., 2005; Richter et al., 2000).

We found rotation-related activity in regions in several clusters within the posterior parietal cortex: the precuneus and the posterior, medial, and anterior parts of the IPS. Several functional subdivisions have been suggested for the posterior parietal cortex (Culham \& Valyear, 2006; Grefkes \& Fink, 2005), but it seems that mental rotation does not selectively recruit one specific parietal area. The parietal node situated in the mIPS appears to correspond to the human PEF or its putative monkey homologue lateral intraparietal (LIP), which is involved in attention and the control of eye movement (Koyama et al., 2004; Berman et al., 1999). The corresponding parietal functional subdivisions of the anterior and posterior segments of IPS are less unequivocal. The parietal activation we found in the aIPS is compatible with the coordinates of a parietal area that has been implicated in mental rotation proficiency (Wolbers et al., 2006) and is in the immediate vicinity of anterior intraparietal, an area involved in tactile and visual object processing (Grefkes \& Fink, 2005). However, it is also anatomically close to VIP, a polymodal motion-sensitive area in the parietal cortex (Bremmer et al., 2001). Finally, the parietal node in the pIPS seems to be too inferior to 
correspond to the orientation-sensitive CIP (Shikata et al., 2001, 2003).

\section{Neural Effects Related to the Perturbation in hV5/MT+}

We were able to perturb activity in hV5/MT + in a directionselective manner by using an MAE.

This technique of perturbation bears resemblance to the more commonly used perturbation technique of TMS but has an important advantage over the transcranial magnetic perturbation. We could change responsiveness of an ensemble of neurons with a specific directional tuning, something that would not be feasible with TMS. In this manner, we could directly assess the effect of this perturbation in $\mathrm{hV} 5 / \mathrm{MT}+$ on other cortical areas. We found that a perturbation in hV5/MT+ led to increased activity in hV5/MT+ itself as well as several regions in the parietal cortex. The results of the MAE localizer experiment confirmed that the perturbation primarily targeted hMT/V5+ because we only found a main effect of MAE in this area but not in the parietal regions that were modulated by the perturbation. Hence, our findings indicate that hMT/V5+ is functionally relevant for visual mental imagery of motion.

The MAE is the result of a bottom-up process (Krekelberg, Boynton, \& van Wezel, 2006), whereas mental rotation requires a top-down process in the form of an imagined stimulus transformation (Mechelli, Price, Friston, \& Ishai, 2004; Goebel, Khorram-Sefat, Muckli, Hacker, \& Singer, 1998). That the site of interaction between these two processes is not restricted to hV5/MT+ but extends into parietal cortex suggests a strong functional coupling between these areas where the parietal cortical areas potentially use input from hV5/MT + to perform the imagined stimulus transformation in mental rotation. Parietal areas LIP and VIP are reciprocally connected with V5/MT+ in monkeys (Lewis \& VanEssen, 2000; Maunsell \& Vanessen, 1983), and the modulated parietal nodes we observed in the respective middle and aIPS potentially correspond to these parietal areas. Furthermore, a series of recent concurrent TMS-fMRI studies demonstrated that TMS of the right PEF has an effect on the BOLD signal in hV5/MT+ when moving stimuli are presented (Ruff et al., 2008, 2009).

So what could be the role of hV5/MT+ in the mental rotation network? On the basis of the parietal nodes that also reflect the perturbation, we propose a hypothetical processing model where hV5/MT+ provides sensory feedback in the form of motion imagery for the ongoing imagined stimulus transformation. Given the presence of both PEF and FEF in the mental rotation network, the transformation potentially relies on eye movements and/or covert shifts of attention because both are supported by these same nodes (Corbetta et al., 1998).

Not only do eye movements have a crucial role in mental imagery (Laeng \& Teodorescu, 2002), in mental rotation they mimic the path of the required rotation (de'Sperati, 2003). PEF might even translate the angular disparity in a transformation plan: At least one study suggests that LIP links behaviorally relevant visual information with motor variables relevant for solving a task in a wide range of circumstances, involving among others goal-directed eye movements (Oristaglio, Schneider, Balan, \& Gottlieb, 2006). The transformation could subsequently generate imagined motion in hV5/MT+ that in turn provides sensory feedback for the ongoing transformation. Integrating sensory feedback with plans to form suitable movements is one of the key functions of parietal cortex. Furthermore, a single-cell study demonstrates that LIP transforms motion direction encoded in hV5/MT+ to more behaviorally relevant representations (Freedman \& Assad, 2006).

\section{Interpretational Issues}

Can our results be explained by differences in attention? Although an MAE has been associated with increased neural activity in hV5/MT + (Tootell, 1995), this neural effect might arise from a lack of control of attention over conditions. Although attentional confounds can explain some of the findings related to MAE, direction-selective adaptation still produces a direction-selective imbalance in hV5/MT+ responses after controlling for the attentional confound (Huk, Ress, \& Heeger, 2001). This is compatible with the findings of single-cell studies who demonstrate that the underlying mechanism of the MAE is probably a combination of (i) a change in the tuning curves of direction-selective neurons that encode the preferred direction of motion and (ii) a disinhibition of neurons that encode the opposite direction of motion (Krekelberg et al., 2006; Kohn \& Movshon, 2003). Moreover, in the experimental design we used, attention is controlled for the following: Not only was there an MAE in both incongruent and congruent trials, subjects were also engaged in a speeded response task in both trial types. Still we found an asymmetry in mental rotation performance, corresponding to the directionselective neuronal imbalance in $\mathrm{hV} 5 / \mathrm{MT}+$ responses. This makes it unlikely that attention can explain the pattern of results we obtained.

Although the neuronal activity in putative FEF was not modulated by the MAE congruency, the observed increased activity during the incongruent condition might be the result of additional eye movements. Both smooth pursuit eye movements and saccades are known to activate hV5/ MT+ and posterior parietal cortex (Petit \& Haxby, 1999). We created a direction-selective imbalance in hV5/MT + neurons while subjects were required to fixate the center of the radial sinusoidal grating. This might have resulted in eye movements in the opposite direction during the ensuing MAE. Adaptation to a moving stimulus can create an oculomotor MAE with both pursuit and saccadic components, but only in the presence of a visual stimulus, suggesting that these eye movements are a perceptual effect (Watamaniuk \& Heinen, 2007). To gain more insight in the role of these potential eye movements, future research where eye movements are controlled for would be of particular interest. 
It remains to be seen whether an incongruent MAE obstructs the rotation of the visual mental image or of the physical visual stimulus that is presented, implying a perceptual effect in the latter case. It could be that because of the MAE, the visual stimulus is perceived to rotate in the wrong direction, subsequently requiring more mental rotation. Although this can at present not be excluded, a recent behavioral experiment is suggestive of the fact that the hV5/MT+ perturbation interferes with the internal representation or mental image rather than with the physical stimulus. In this study, which used the same paradigm as the current study, the mental rotation stimuli were not kept on screen but flashed for a brief period (180 msec). Although none of the subjects reported an MAE, there was an equally robust MAE congruency effect (R. Seurinck, F.P. de Lange, R. Achten, and G. Vingerhoets, unpublished observations), in line with an interference of the visual mental image.

\section{Conclusions}

We have shown that hV5/MT+ is necessary for mental imagery of visual motion: The activity in this area increased with increasing mental rotation, and a direction-selective perturbation of neurons in this area affected behavioral performance as well as the activity in this area. Moreover, several regions in the posterior parietal cortex were affected by the perturbation in hV5/MT+.

Our findings suggest that mental imagery of visual motion relies on an interaction between $\mathrm{hV} 5 / \mathrm{MT}+$ and parietal cortex. This paradigm therefore seems well suited to investigate the dynamical interactions between these nodes. Future studies, looking at effective connectivity using dynamic causal modeling (Friston, Harrison, \& Penny, 2003), are necessary to shed more light on the exact dynamics of these interactions.

\section{Acknowledgments}

This work was supported by grant no. G.0158.02 of the Fund for Scientific Research, Belgium. The authors thank Ivan Toni for his helpful comments and suggestions.

Reprint requests should be sent to Ruth Seurinck, Department of Experimental Psychology, H. Dunantlaan 2, B-9000 Ghent, Belgium, or via e-mail: ruth.seurinck@ugent.be.

\section{REFERENCES}

Amiez, C., Kostopoulos, P., Champod, A. S., \& Petrides, M. (2006). Local morphology predicts functional organization of the dorsal premotor region in the human brain. Journal of Neuroscience, 26, 2724-2731.

Anand, S., Olson, J. D., \& Hotson, J. R. (1998). Tracing the timing of human analysis of motion and chromatic signals from occipital to temporo-parieto-occipital cortex: A transcranial magnetic stimulation study. Vision Research, 38, 2619-2627.

Beckers, G., \& Zeki, S. (1995). The consequences of inactivating areas V1 and V5 on visual-motion perception. Brain, 118, 49-60.
Berman, R. A., Colby, C. L., Genovese, C. R., Voyvodic, J. T., Luna, B., Thulborn, K. R., et al. (1999). Cortical networks subserving pursuit and saccadic eye movements in humans: An fMRI study. Human Brain Mapping, 8, 209-225.

Bremmer, F., Schlack, A., Shah, N. J., Zafiris, O., Kubischik, M., Hoffmann, K. P., et al. (2001). Polymodal motion processing in posterior parietal and promotor cortex: A human fMRI study strongly implies equivalencies between humans and monkeys. Neuron, 29, 287-296.

Brett, M., Anton, J. L., Valabregue, R., \& Poline, J. B. (2002). Region of interest analysis using an SPM toolbox [Abstract]. Presented at the 8th International Conference on Functional Mapping of the Human Brain, June 2-6, 2002, Sendai, Japan. Available on CD-ROM in Neuroimage, 16(2).

Corbetta, M., Akbudak, E., Conturo, T. E., Snyder, A. Z., Ollinger, J. M., Drury, H. A., et al. (1998). A common network of functional areas for attention and eye movements. Neuron, 21, 761-773.

Culham, J. C., \& Valyear, K. F. (2006). Human parietal cortex in action. Current Opinion in Neurobiology, 16, 205-212.

d'Alfonso, A. A. L., van Honk, J., Schutter, D., Caffe, A. R., Postma, A., \& de Haan, E. H. F. (2002). Spatial and temporal characteristics of visual motion perception involving V5 visual cortex. Neurological Research, 24, 266-270.

de Lange, F. P., Hagoort, P., \& Toni, I. (2005). Neural topography and content of movement representations. Journal of Cognitive Neuroscience, 17, 97-112.

de'Sperati, C. (2003). Precise oculomotor correlates of visuospatial mental rotation and circular motion imagery. Journal of Cognitive Neuroscience, 15, 1244-1259.

Dumoulin, S. O., Bittar, R. G., Kabani, N. J., Baker, C. L., Le Goualher, G., Pike, G. B., et al. (2000). A new anatomical landmark for reliable identification of human area V5/MT: A quantitative analysis of sulcal patterning. Cerebral Cortex, 10, 454-463.

Duvernoy, H. M., Cabanis, E. A., \& Vannson, J. L. (1991). The buman brain: Surface three-dimensional sectional anatomy and MRI. Wien: Springer-Verlag.

Ecker, C., Brammer, M. J., David, A. S., \& Williams, S. C. (2006). Time-resolved fMRI of mental rotation revisited-dissociating visual perception from mental rotation in female subjects. Neuroimage, 32, 432-444.

Eickhoff, S. B., Stephan, K. E., Mohlberg, H., Grefkes, C., Fink, G. R., Amunts, K., et al. (2005). A new SPM toolbox for combining probabilistic cytoarchitectonic maps and functional imaging data. Neuroimage, 25, 1325-1335.

Freedman, D. J., \& Assad, J. A. (2006). Experience-dependent representation of visual categories in parietal cortex. Nature, 443, 85-88.

Friston, K. J., Ashburner, J., Frith, C. D., Poline, J. B., Heather, J. D., \& Frackowiak, R. S. J. (1995). Spatial registration and normalization of images. Human Brain Mapping, 3, 165-189.

Friston, K. J., Harrison, L., \& Penny, W. (2003). Dynamic causal modelling. Neuroimage, 19, 1273-1302.

Friston, K. J., Holmes, A. P., Price, C. J., Buchel, C., \& Worsley, K. J. (1999). Multisubject fMRI studies and conjunction analyses. Neuroimage, 10, 385-396.

Friston, K. J., Holmes, A. P., \& Worsley, K. J. (1999). How many subjects constitute a study? Neuroimage, 10, 1-5.

Friston, K. J., Holmes, A. P., Worsley, K. J., Poline, J. P., Frith, C. D., \& Frackowiak, R. S. J. (1994). Statistical parametric maps in functional imaging: A general linear approach. Human Brain Mapping, 2, 189-210.

Friston, K. J., Rotshtein, P., Geng, J. J., Sterzer, P., \& Henson, R. N. (2006). A critique of functional localisers. Neuroimage, 30, 1077-1087.

Goebel, R., Khorram-Sefat, D., Muckli, L., Hacker, H., \& Singer, W. (1998). The constructive nature of vision: Direct evidence 
from functional magnetic resonance imaging studies of apparent motion and motion imagery. European Journal of Neuroscience, 10, 1563-1573.

Grefkes, C., \& Fink, G. R. (2005). The functional organization of the intraparietal sulcus in humans and monkeys. Journal of Anatomy, 207, 3-17.

Heil, M., Bajric, J., Rosler, F., \& Hennighausen, E. (1997). A rotation aftereffect changes both the speed and the preferred direction of mental rotation. Journal of Experimental Psychology: Human Perception and Performance, 23, 681-692.

Hotson, J. R., \& Anand, S. (1999). The selectivity and timing of motion processing in human temporo-parieto-occipital and occipital cortex: A transcranial magnetic stimulation study. Neuropsychologia, 37, 169-179.

Huk, A. C., Ress, D., \& Heeger, D. J. (2001). Neuronal basis of the motion aftereffect reconsidered. Neuron, 32, 161-172.

Klein, I., Dubois, J., Mangin, J. F., Kherif, F., Flandin, G., Poline, J. B., et al. (2004). Retinotopic organization of visual mental images as revealed by functional magnetic resonance imaging. Cognitive Brain Research, 22, 26-31.

Kohn, A., \& Movshon, J. A. (2003). Neuronal adaptation to visual motion in area MT of the macaque. Neuron, 39, 681-691.

Kosslyn, S. M. (1994). Image and brain: The resolution of the imagery debate. Cambridge, MA: MIT Press.

Koyama, M., Hasegawa, I., Osada, T., Adachi, Y., Nakahara, K., \& Miyashita, Y. (2004). Functional magnetic resonance imaging of macaque monkeys performing visually guided saccade tasks: Comparison of cortical eye fields with humans. Neuron, 41, 795-807.

Krekelberg, B., Boynton, G. M., \& van Wezel, R. J. A. (2006). Adaptation: From single cells to BOLD signals. Trends in Neurosciences, 29, 250-256.

Laeng, B., \& Teodorescu, D. S. (2002). Eye scanpaths during visual imagery reenact those of perception of the same visual scene. Cognitive Science, 26, 207-231.

Lewis, J. W., \& VanEssen, D. C. (2000). Corticocortical connections of visual, sensorimotor, and multimodal processing areas in the parietal lobe of the macaque monkey. Journal of Comparative Neurology, 428, 112-137.

Maunsell, J. H. R., \& Vanessen, D. C. (1983). The connections of the middle temporal visual area (Mt) and their relationship to a cortical hierarchy in the macaque monkey. Journal of Neuroscience, 3, 2563-2586.

Mayka, M. A., Corcos, D. M., Leurgans, S. E., \& Vaillancourt, D. E. (2006). Three-dimensional locations and boundaries of motor and premotor cortices as defined by functional brain imaging: A meta-analysis. Neuroimage, 31, 1453-1474.

Mechelli, A., Price, C. J., Friston, K. J., \& Ishai, A. (2004). Where bottom-up meets top-down: Neuronal interactions during perception and imagery. Cerebral Cortex, 14, 1256-1265.

Mourao-Miranda, J., Ecker, C., Sato, J. R., \& Brammer, M. (2009). Dynamic changes in the mental rotation network revealed by pattern recognition analysis of fMRI data. Journal of Cognitive Neuroscience, 21, 890-904.

Newsome, W. T., \& Pare, E. B. (1988). A selective impairment of motion perception following lesions of the middle temporal visual area (Mt). Journal of Neuroscience, 8, 2201-2211.

Oristaglio, J., Schneider, D. M., Balan, P. F., \& Gottlieb, J. (2006). Integration of visuospatial and effector information during symbolically cued limb movements in monkey lateral intraparietal area. Journal of Neuroscience, 26, 8310-8319.

Parsons, L. M., Fox, P. T., Downs, J. H., Glass, T., Hirsch, T. B., Martin, C. C., et al. (1995). Use of implicit motor imagery for visual shape-discrimination as revealed by Pet. Nature, 375, 54-58.

Petit, L., \& Haxby, J. V. (1999). Functional anatomy of pursuit eye movements in humans as revealed by fMRI. Journal of Neurophysiology, 82, 463-471.
Pitzalis, S., Galletti, C., Huang, R. S., Patria, F., Committeri, G., Galati, G., et al. (2006). Wide-field retinotopy defines human cortical visual area V6. Journal of Neuroscience, 26, 7962-7973.

Richter, W., Somorjai, R., Summers, R., Jarmasz, M., Menon, R. S., Gati, J. S., et al. (2000). Motor area activity during mental rotation studied by time-resolved single-trial fMRI. Journal of Cognitive Neuroscience, 12, 310-320.

Ruff, C. C., Bestmann, S., Blankenburg, F., Bjoertomt, O., Josephs, O., Weiskopf, N., et al. (2008). Distinct causal influences of parietal versus frontal areas on human visual cortex: Evidence from concurrent TMS-fMRI. Cerebral Cortex, 18, 817-827.

Ruff, C. C., Blankenburg, F., Bjoertomt, O., Bestmann, S., Weiskopf, N., \& Driver, J. (2009). Hemispheric differences in frontal and parietal influences on human occipital cortex: Direct confirmation with concurrent TMS-fMRI. Journal of Cognitive Neuroscience, 21, 1146-1161.

Shepard, R. N., \& Metzler, J. (1971). Mental rotation of 3-dimensional objects. Science, 171, 701-703.

Shikata, E., Hamzei, F., Glauche, V., Knab, R., Dettmers, C., Weiller, C., et al. (2001). Surface orientation discrimination activates caudal and anterior intraparietal sulcus in humans: An event-related fMRI study. Journal of Neurophysiology, 85, 1309-1314.

Shikata, E., Hamzei, F., Glauche, V., Koch, M., Weiller, C., Binkofski, F., et al. (2003). Functional properties and interaction of the anterior and posterior intraparietal areas in humans. European Journal of Neuroscience, 17, 1105-1110.

Slotnick, S. D., Thompson, W. L., \& Kosslyn, S. M. (2005). Visual mental imagery induces retinotopically organized activation of early visual areas. Cerebral Cortex, 15, 1570-1583.

Sunaert, S., Van Hecke, P., Marchal, G., \& Orban, G. A. (1999). Motion-responsive regions of the human brain. Experimental Brain Research, 127, 355-370.

Tootell, R. (1995). Visual-motion aftereffect in human cortical area MT revealed by functional magnetic-resonance-imaging. Nature, 375, 139-141.

Tootell, R. B. H., Reppas, J. B., Kwong, K. K., Malach, R., Born, R. T., Brady, T. J., et al. (1995). Functional-analysis of human Mt and related visual cortical areas using magneticresonance-imaging. Journal of Neuroscience, 15, 3215-3230.

Van Wezel, R. J. A., \& Britten, K. H. (2002). Multiple uses of visual motion. The case for stability in sensory cortex. Neuroscience, 111, 739-759.

Walsh, V., Ellison, A., Battelli, L., \& Cowey, A. (1998). Task-specific impairments and enhancements induced by magnetic stimulation of human visual area V5. Philosophical Transactions of the Royal Society of London, Series B, Biological Sciences, 265, 537-543.

Watamaniuk, S. N. J., \& Heinen, S. J. (2007). Storage of an oculomotor motion aftereffect. Vision Research, 47, 466-473.

Watson, J. D. G., Myers, R., Frackowiak, R. S. J., Hajnal, J. V., Woods, R. P., Mazziotta, J. C., et al. (1993). Area-V5 of the human brain-Evidence from a combined study using positron emission tomography and magnetic-resonance-imaging. Cerebral Cortex, 3, 79-94.

Wolbers, T., Schoell, E. D., \& Buchel, C. (2006). The predictive value of white matter organization in posterior parietal cortex for spatial visualization ability. Neuroimage, 32, 1450-1455.

Worsley, K. J., Marrett, S., Neelin, P., Vandal, A. C., Friston, K. J., \& Evans, A. C. (1996). A unified statistical approach for determining significant signals in images of cerebral activation. Human Brain Mapping, 4, 58-73.

Zacks, J. M. (2008). Neuroimaging studies of mental rotation: A meta-analysis and review. Journal of Cognitive Neuroscience, 20, 1-19. 